\title{
The influence of marital status on the survival of patients with esophageal cancer: a population-based, propensity-matched study
}

\author{
Qing-Wei Zhang ${ }^{1, *}$, Xiao-Lu Lin ${ }^{1,5 *}$, Chi-Hao Zhang ${ }^{2, *}$, Chen-Yue Tang ${ }^{3, *}$, Xin-Tian \\ Zhang $^{1, *}$, La-Mei Teng ${ }^{1,4, *}$, Zhi-Zheng Ge ${ }^{1}$ and Xiao-Bo Li ${ }^{1}$ \\ ${ }^{1}$ Division of Gastroenterology and Hepatology, Key Laboratory of Gastroenterology and Hepatology, Ministry of Health, Renji \\ Hospital, School of Medicine, Shanghai Jiao Tong University, Shanghai Institute of Digestive Disease, Shanghai 200001, \\ China \\ ${ }^{2}$ Department of General Surgery, Shanghai Ninth People's Hospital, School of Medicine, Shanghai Jiao Tong University, \\ Baoshan 201999, Shanghai, China \\ ${ }^{3}$ Department of Gastroenterology, Ruijin Hospital, Shanghai Jiao Tong University School of Medicine, Shanghai 200000, China \\ ${ }^{4}$ Division of Gastroenterology and Hepatology, Liqun Clinical Medicine College, The Second Military Medical University, Liqun \\ Hospital, Shanghai 200001, China \\ ${ }^{5}$ Department of Digestive Endoscopy, Provincial Clinic Medical College, Fujian Medical University, Fujian Provincial Hospital, \\ Fuzhou 350001, China \\ *These authors have contributed equally to this work \\ Correspondence to: Zhi-Zheng Ge, email: zhizhengge@aliyun.com \\ Xiao-Bo Li, email: Ixb_1969@163.com \\ Keywords: esophageal cancer, marital status, SEER, OS, CSS \\ Received: February 22, $2017 \quad$ Accepted: April 04, $2017 \quad$ Published: July 22, 2017 \\ Copyright: Zhang et al. This is an open-access article distributed under the terms of the Creative Commons Attribution License 3.0 (CC BY 3.0), \\ which permits unrestricted use, distribution, and reproduction in any medium, provided the original author and source are credited.
}

\section{ABSTRACT}

Background and aims: Multiple studies have shown that marital status is associated with the survival of various types of cancer patients. However, there has not been adequate evidence of the association between marital status and the survival of patients with esophageal cancer (EC). We aimed to investigate the effect of marital status on survival of EC patients.

Methods: We identified 15,598 EC patients from the Surveillance, Epidemiology, and End Results (SEER) database. Meanwhile, propensity scores for marital status, which were calculated for each patient using a nonparsimonious multivariable logistic regression model, were used to match 6,319 unmarried patients with 9,279 married patients. We performed Kaplan-Meier analysis and multivariate Cox regression to analyze the association between marital status and the overall survival (OS) and EC cause-specific survival (CSS) of EC patients before matching and after matching.

Results: We matched 2,986 unmarried patients with 2,986 married patients. Unmarried patients had poorer OS than married patients before matching (hazard ratio [HR]: 1.22; 95\% confidence interval [CI]: 1.18-1.27; $P<0.0001$ ) and after matching (HR: 1.20; 95\% CI: 1.13-1.27; $P<0.0001$ ) and poorer CSS than married patients before matching (HR: 1.21; 95\% CI: 1.16-1.26; $P<0.0001$ ) and after matching (HR: 1.17; 95\% CI: 1.10-1.24; P < 0.0001). Further analysis showed that among different unmarried patients, widowed patients had the poorest OS (HR: 1.46; 95\% CI: 1.38-1.55; P < 0.0001) and CSS (HR: 1.43; 95\% CI: 1.34-1.52; P < 0.0001) compared with married patients.

Conclusions: Unmarried EC patients had poorer survival rates than married EC patients. Meanwhile, widowed patients with EC had the highest risk of death compared with single, married, and divorced patients. 


\section{INTRODUCTION}

Esophageal cancer (EC) is one of ten leading causes of cancer-related deaths, with 16,940 new cases and 15,690 deaths in the US [1]. Although the management and treatment of EC has been improved in recent years, the survival of patients with EC is still poor, with a five-year survival rate of $22 \%$ [2]. Several clinical characteristics have proved to be associated with poor patient survival, including tumor grade, tumor stage at diagnosis, and whether surgery or adjuvant therapy was performed. Socioeconomic factors are also likely to contribute to the survival of patients with $\mathrm{EC}$, which is an association that has been demonstrated in other cancers [3-7].

One socioeconomic factor, marital status, contributes to better health with various diseases, including malignancies [8]. Previous studies have demonstrated that marital status played an important role in patients' survival of various cancers, such as liver, gastric, colorectal, and pancreatic cancers [4-7]. However, conclusions about the contribution of marital status to patients' survival have contradicted each other $[3,9,10]$. One population-based nationwide Swedish cohort study that included patients with gastric cancer or EC found that unmarried patients had poorer survival rates than married patients [9]. On the other hand, a prospective populationbased cohort that included postsurgery EC patients from all Swedish hospitals investigated the role of marital status in EC survival but found that unmarried patients did not have poorer survival rates than married patients [10]. In a large population-based study that used data from the Surveillance, Epidemiology, and End Results (SEER) database, the authors used the Cox proportional hazards multivariable regression while adjusting for age, sex, race, income, residence, nodal stage, tumor stage, educational level, metastatic status, and use of definitive therapy to analyze the role of marital status in the ten leading cancers that cause cancer-related deaths, including EC, concluding that unmarried patients had a higher risk death from these cancers [3].

However, marital statuses have changed in recent years [11]. In addition, delayed diagnosis, rejection of therapy, and a lack of social support could contribute to poor prognosis of cancers, which could all also be influenced by marital status $[12,13]$. Therefore, the potential association between marital status and EC survival is still unclear and should be analyzed.

We conducted a study that involved a large population consisting of patients diagnosed between 2004 and 2012 and that included information about tumor grade, tumor histology, location of the EC, and more detailed therapy information. In this study, which was based on Aizer's study [3], we further explored whether different marital statuses could affect the survival of EC patients. This study employed propensity score matching (PSM), which is an effective tool to reduce selection and residual biases. Furthermore, we used Cox proportional hazards multivariable regression to reanalyze the role of marital status in the survival of EC patients in the matched cohort using PSM with a balance of studied exposure variables between married and unmarried patients.

\section{RESULTS}

\section{Patient characteristics}

In total, 15,598 patients with EC who met our inclusion criteria, including 9,279 (59.49\%) married patients and 6,319 (40.51\%) unmarried patients, were identified in the SEER database. Of the unmarried patients, 1,971 (12.62\%) were divorced, 2,592 (16.62\%) were single, and $1,756(11.26 \%)$ were widowed. Table 1 describes the clinicopathological characteristics of the patients with different marital statuses.

As shown in Table 1, compared with the male patients, more female patients tended to be unmarried. Among the widowed patients, 906 (51.59\%) were female, compared with proportion of females in patients with other marital statuses. With respect to the patients' race, Black patients were more likely to be unmarried than White patients and patients of other races. Compared with married patients, unmarried patients were more likely to receive no surgery or radiotherapy, to have esophageal adenocarcinoma (EAC), and to have cancers located in the middle third of the esophagus. However, there seemed to be little difference in the patients' TNM stage, which had a standard difference (SD) of 0.049 , and tumor grade (SD: 0.079). The detailed clinicopathological characteristics of the patients with different marital statuses are shown in Table 1.

\section{The effects of marital status on overall survival and cause-specific survival in the unmatched 15,598-patient cohort with esophageal cancer}

Kaplan-Meier curves were used to evaluate the overall survival (OS) rate of EC patients (see Figure 1A). As shown in Figure 1A, unmarried patients had poorer prognoses (hazard ratio [HR]: 1.32; 95\% confidence interval [CI]: $1.27-1.37 ; \mathrm{P}<0.0001)$ than married patients according to the Cox proportional hazards univariate regression model. After controlling the patients' baseline characteristics, including age, sex, race, therapy, TNM stage, tumor grade, tumor location, and tumor histology, unmarried patients still had poorer prognoses (HR: 1.22; 95\% CI: $1.18-1.27 ; \mathrm{P}<0.0001)$ than married patients.

The cause-specific survival rates (CSS) of EC patients were also plotted using Kaplan-Meier curves. As shown in Figure 1B, compared with being married, being unmarried contributed to poor prognoses (HR: 1.30 ; 95\% CI: $1.25-1.35 ; \mathrm{P}<0.0001)$ according to the Cox proportional hazards univariate regression model and 
Table 1: Characteristics of patients with esophageal carcinomas in SEER database

\begin{tabular}{|c|c|c|c|c|c|c|}
\hline \multirow[t]{2}{*}{ Characteristics } & Total & Married & Unmarried & Divorced & Single & Widowed \\
\hline & $15598(100 \%)$ & $9279(59.49 \%)$ & $6319(40.51 \%)$ & $1971(12.64 \%)$ & $2592(16.62 \%)$ & $1756(11.26 \%)$ \\
\hline \multicolumn{7}{|l|}{ Sex } \\
\hline Male & $\begin{array}{c}12581 \\
(80.66 \%)\end{array}$ & $8093(87.22 \%)$ & $4488(71.02 \%)$ & $1555(78.89 \%)$ & $2083(80.36 \%)$ & $850(48.41 \%)$ \\
\hline Female & $3017(19.34 \%)$ & $1186(12.78 \%)$ & $1831(28.98 \%)$ & $416(21.11 \%)$ & $509(19.64 \%)$ & $906(51.59 \%)$ \\
\hline \multicolumn{7}{|l|}{ Race } \\
\hline White & $\begin{array}{c}13241 \\
(84.89 \%)\end{array}$ & $8297(89.42 \%)$ & $4944(78.24 \%)$ & $1629(82.65 \%)$ & $1879(72.49 \%)$ & $1436(81.78 \%)$ \\
\hline Black & $1583(10.15 \%)$ & $480(5.17 \%)$ & $1103(17.46 \%)$ & $273(13.85 \%)$ & $609(23.50 \%)$ & $221(12.59 \%)$ \\
\hline Other race & $774(4.96 \%)$ & $502(5.41 \%)$ & $272(4.30 \%)$ & $69(3.50 \%)$ & $104(4.01 \%)$ & $99(5.64 \%)$ \\
\hline \multicolumn{7}{|l|}{ Age } \\
\hline$<40$ & $216(1.38 \%)$ & $103(1.11 \%)$ & $113(1.79 \%)$ & $16(0.81 \%)$ & $95(3.67 \%)$ & $2(0.11 \%)$ \\
\hline $41-55$ & $2985(19.14 \%)$ & $1604(17.29 \%)$ & $1381(21.85 \%)$ & $466(23.64 \%)$ & $863(33.29 \%)$ & $52(2.96 \%)$ \\
\hline $56-70$ & $7233(46.37 \%)$ & $4565(49.20 \%)$ & $2668(42.22 \%)$ & $1062(53.88 \%)$ & $1175(45.33 \%)$ & $431(24.54 \%)$ \\
\hline $71-85$ & $4416(28.31 \%)$ & $2687(28.96 \%)$ & $1729(27.36 \%)$ & $399(20.24 \%)$ & $396(15.28 \%)$ & $934(53.19 \%)$ \\
\hline$>85$ & $748(4.80 \%)$ & $320(3.45 \%)$ & $428(6.77 \%)$ & $28(1.42 \%)$ & $63(2.43 \%)$ & $337(19.19 \%)$ \\
\hline \multicolumn{7}{|l|}{ Histology } \\
\hline ESCC & $4725(30.29 \%)$ & $2231(24.04 \%)$ & $2494(39.47 \%)$ & $696(35.31 \%)$ & $1071(41.32 \%)$ & 727 (41.40\%) \\
\hline EAC & $9530(61.10 \%)$ & $6193(66.74 \%)$ & $3337(52.81 \%)$ & $1119(56.77 \%)$ & $1348(52.00 \%)$ & $870(49.54 \%)$ \\
\hline Others & $1343(8.61 \%)$ & $855(9.21 \%)$ & $488(7.72 \%)$ & $156(7.91 \%)$ & $173(6.67 \%)$ & $159(9.05 \%)$ \\
\hline \multicolumn{7}{|l|}{ Grade } \\
\hline $\begin{array}{c}\text { Well } \\
\text { differentiated }\end{array}$ & $844(5.41 \%)$ & $507(5.46 \%)$ & $337(5.33 \%)$ & $100(5.07 \%)$ & $136(5.25 \%)$ & $101(5.75 \%)$ \\
\hline $\begin{array}{l}\text { Moderately } \\
\text { differentiated }\end{array}$ & $6276(40.24 \%)$ & $3592(38.71 \%)$ & $2684(42.48 \%)$ & $834(42.31 \%)$ & $1120(43.21 \%)$ & $730(41.57 \%)$ \\
\hline $\begin{array}{c}\text { Poorly } \\
\text { differentiated }\end{array}$ & $8172(52.39 \%)$ & $5002(53.91 \%)$ & $3170(50.17 \%)$ & $1009(51.19 \%)$ & $1290(49.77 \%)$ & $871(49.60 \%)$ \\
\hline Undifferentiated & $306(1.96 \%)$ & $178(1.92 \%)$ & $128(2.03 \%)$ & $28(1.42 \%)$ & $46(1.77 \%)$ & $54(30.75 \%)$ \\
\hline \multicolumn{7}{|l|}{ Location } \\
\hline $\begin{array}{l}\text { Upper third of } \\
\text { esophagus }\end{array}$ & $1053(6.75 \%)$ & $481(5.18 \%)$ & $572(9.05 \%)$ & $167(8.47 \%)$ & $245(9.45 \%)$ & $160(9.11 \%)$ \\
\hline $\begin{array}{l}\text { Middle third of } \\
\text { esophagus }\end{array}$ & $3186(20.43 \%)$ & $1595(17.19 \%)$ & $1591(25.18 \%)$ & $459(23.29 \%)$ & $655(25.27 \%)$ & $477(27.16 \%)$ \\
\hline $\begin{array}{l}\text { Lower third of } \\
\text { esophagus }\end{array}$ & $\begin{array}{c}11359 \\
(72.82 \%)\end{array}$ & $7203(77.63 \%)$ & $4156(65.77 \%)$ & 1345 (68.24\%) & $1692(65.28 \%)$ & $1119(63.72 \%)$ \\
\hline
\end{tabular}

\section{TNM Stage}

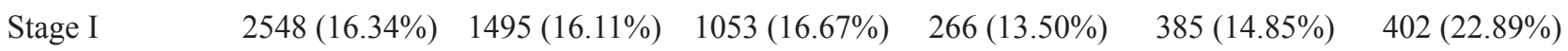

Stage II $\quad 3317(21.27 \%) \quad 1949(21.00 \%) \quad 1368(21.65 \%) \quad 428(21.71 \%) \quad 524(20.22 \%) \quad 416(23.69 \%)$

Stage III $\quad 3733(23.93 \%) \quad 2299(24.78 \%) \quad 1434(22.69 \%) \quad 487(24.71 \%) \quad 595(22.96 \%) \quad 352(20.04 \%)$

(Continued) 
Table 1: (Continued) Characteristics of patients with esophageal carcinomas in SEER database

\begin{tabular}{|c|c|c|c|c|c|c|}
\hline \multirow[t]{2}{*}{ Characteristics } & Total & Married & Unmarried & Divorced & Single & Widowed \\
\hline & $15598(100 \%)$ & $9279(59.49 \%)$ & $6319(40.51 \%)$ & $1971(12.64 \%)$ & $2592(16.62 \%)$ & $1756(11.26 \%)$ \\
\hline Stage IV & $6000(38.47 \%)$ & $3536(38.11 \%)$ & $2464(38.99 \%)$ & $790(40.08 \%)$ & $1088(41.98 \%)$ & $586(33.37 \%)$ \\
\hline \multicolumn{7}{|l|}{ Therapy } \\
\hline $\begin{array}{l}\text { No surgery or } \\
\text { radiotherapy }\end{array}$ & $\begin{array}{c}10247 \\
(65.69 \%)\end{array}$ & $5608(60.44 \%)$ & $4639(73.41 \%)$ & $1371(69.56 \%)$ & $1854(71.53 \%)$ & $1414(80.52 \%)$ \\
\hline Only surgery & $2107(13.51 \%)$ & $1393(15.01 \%)$ & $714(11.30 \%)$ & $224(11.36 \%)$ & $319(12.31 \%)$ & $171(9.74 \%)$ \\
\hline $\begin{array}{l}\text { Only } \\
\text { radiotherapy }\end{array}$ & $359(2.30 \%)$ & $224(2.41 \%)$ & $135(2.14 \%)$ & $45(2.28 \%)$ & $62(2.39 \%)$ & $28(1.59 \%)$ \\
\hline $\begin{array}{l}\text { Surgery and } \\
\text { radiotherapy }\end{array}$ & $2885(18.50 \%)$ & $2054(22.14 \%)$ & $831(13.15 \%)$ & $331(16.79 \%)$ & 357 (13.77\%) & $143(8.14 \%)$ \\
\hline
\end{tabular}

SEER=surveillance, epidemiology and end results; $\mathrm{ESCC}=$ esophageal squamous cell carcinoma; $\mathrm{EAC}=$ esophageal adenocarcinoma; $\mathrm{TNM}=$ tumor, node and metastasis.

even when controlling confounding factors using the Cox proportional hazards multivariate regression model (HR: $1.21 ; 95 \%$ CI: $1.16-1.26$; P $<0.0001$ ).

In addition, age, sex, race, tumor grade, tumor stage, tumor location, and type of therapy were validated as independent prognosis factors for OS and CSS in the multivariate Cox analyses. The detailed description of each prognosis factor is shown in Table 2 .

A

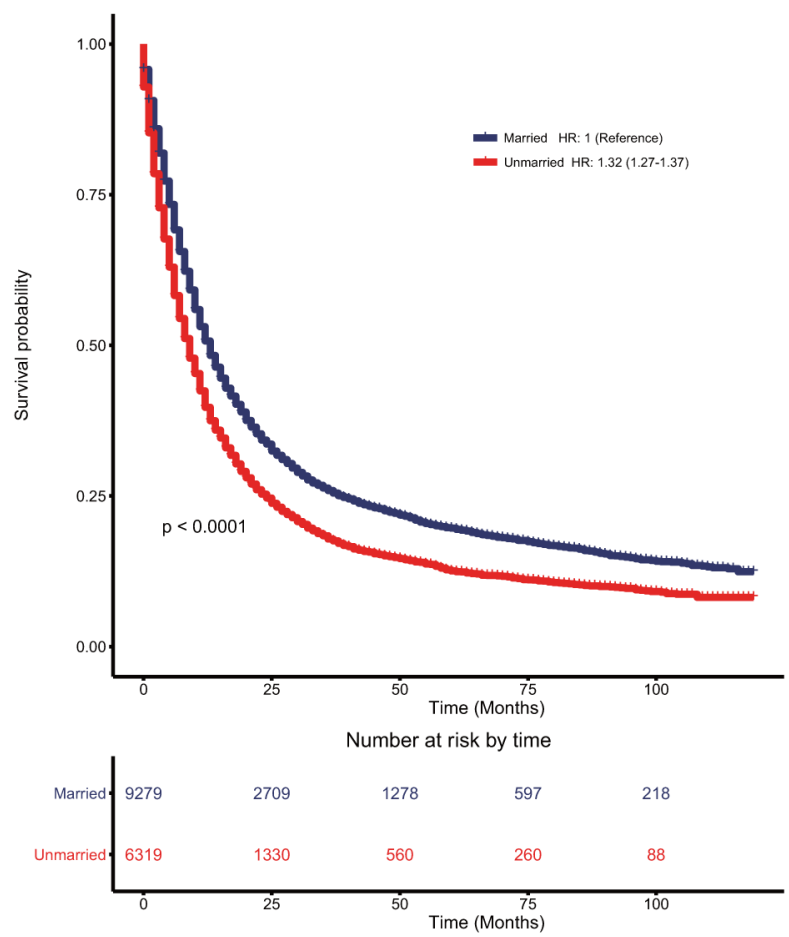

The effects of different unmarried statuses on overall survival and cause-specific survival in the unmatched 15,598-patient cohort with esophageal cancer

To explore whether different unmarried statuses contributed to poorer prognoses than being married, we divided unmarried patients into three subgroups:

B

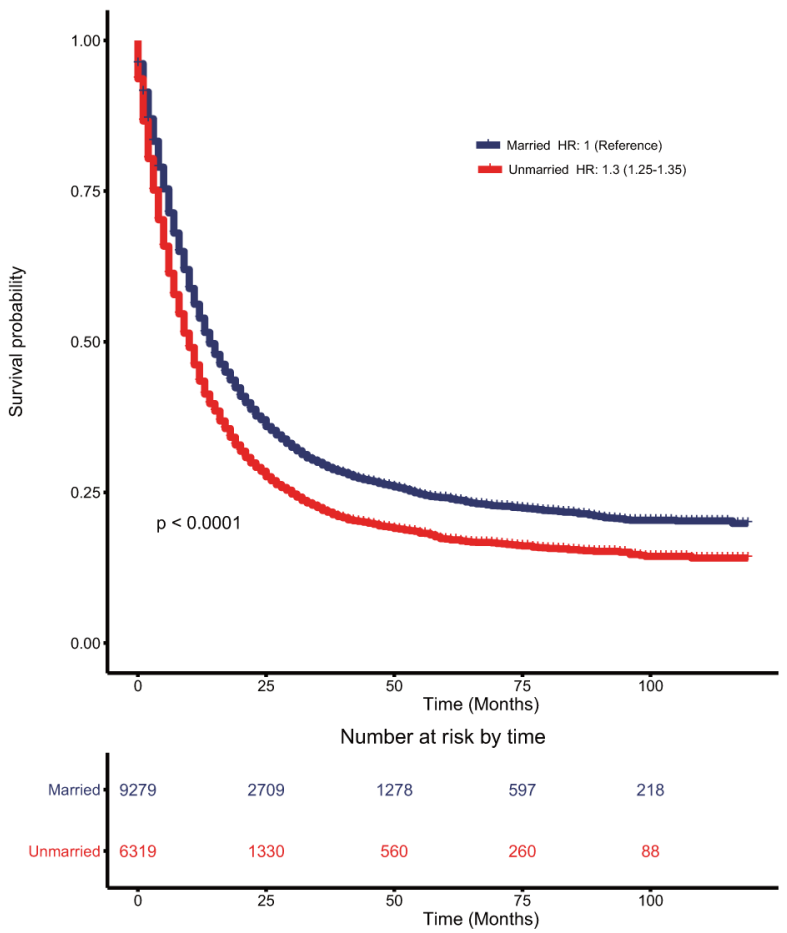

Figure 1: Kaplan-Meier survival plots of the 15,558 patients with esophageal cancers according to marital status (A) overall survival; (B) esophageal cancer cause-specific survival. 
Table 2: Univariate and multivariate Cox regression analysis for evaluating the influence of marital status on OS and esophageal cancer CSS in 15598 unmatched cohort with esophageal cancer in SEER database

\begin{tabular}{|c|c|c|c|c|c|c|c|c|}
\hline \multirow[t]{3}{*}{ Variable } & \multicolumn{4}{|c|}{ OS } & \multicolumn{4}{|c|}{ CSS } \\
\hline & \multicolumn{2}{|c|}{ Univariate analysis } & \multicolumn{2}{|c|}{ Multivariate analysis } & \multicolumn{2}{|c|}{ Univariate analysis } & \multicolumn{2}{|c|}{ Multivariate analysis } \\
\hline & HR $(95 \%$ CI $)$ & $\mathbf{P}$ & HR $(95 \%$ CI $)$ & $\mathbf{P}$ & HR $(95 \%$ CI $)$ & $\mathbf{P}$ & HR $(95 \%$ CI $)$ & $\mathbf{P}$ \\
\hline \multicolumn{9}{|l|}{ Marriage } \\
\hline Married & Reference & & Reference & & Reference & & Reference & \\
\hline Unmarried & $1.28(1.24-1.33)$ & $<0.001$ & $1.22(1.18-1.27)$ & $<0.001$ & $1.26(1.21-1.31)$ & $<0.001$ & $1.21(1.16-1.26)$ & $<0.001$ \\
\hline \multicolumn{9}{|l|}{ Sex } \\
\hline Male & Reference & & Reference & & Reference & & Reference & \\
\hline Female & $0.99(0.95-1.04)$ & 0.854 & $0.86(0.82-0.90)$ & $<0.001$ & $1.00(0.95-1.05)$ & 0.950 & $0.88(0.84-0.93)$ & $<0.001$ \\
\hline \multicolumn{9}{|l|}{ Race } \\
\hline White & Reference & & Reference & & Reference & & Reference & \\
\hline Black & $1.32(1.24-1.40)$ & $<0.001$ & $1.16(1.09-1.24)$ & $<0.001$ & $1.30(1.22-1.38)$ & $<0.001$ & $1.15(1.07-1.23)$ & $<0.001$ \\
\hline Other race & $1.01(0.93-1.10)$ & 0.763 & $0.94(0.86-1.02)$ & 0.154 & $1.01(0.92-1.11)$ & 0.823 & $0.94(0.86-1.03)$ & 0.218 \\
\hline \multicolumn{9}{|l|}{ Age } \\
\hline $\begin{array}{l}(<40) \text { vs }(41-55) \text { vs }(56-70) \text { vs } \\
(71-85) \text { vs }(>85)\end{array}$ & $1.21(1.18-1.24)$ & $<0.001$ & $1.19(1.16-1.22)$ & $<0.001$ & $1.16(1.14-1.19)$ & $<0.001$ & $1.16(1.14-1.19)$ & $<0.001$ \\
\hline \multicolumn{9}{|l|}{ Histology } \\
\hline ESCC & Reference & & Reference & & Reference & & Reference & \\
\hline EAC & $0.84(0.81-0.88)$ & $<0.001$ & $0.99(0.93-1.05)$ & 0.649 & $0.86(0.82-0.9)$ & $<0.001$ & $0.86(0.82-0.9)$ & $<0.001$ \\
\hline Others & $1.14(1.06-1.22)$ & $<0.001$ & $1.14(1.05-1.24)$ & 0.001 & $1.17(1.10-1.27)$ & $<0.001$ & $1.17(1.10-1.27)$ & $<0.001$ \\
\hline \multicolumn{9}{|l|}{ Grade } \\
\hline I vs II vs III vs IV & $1.30(1.27-1.34)$ & $<0.001$ & $1.17(1.14-1.21)$ & $<0.001$ & $1.36(1.31-1.40)$ & $<0.001$ & $1.21(1.17-1.25)$ & $<0.001$ \\
\hline \multicolumn{9}{|l|}{ Location } \\
\hline Upper third of esophagus & Reference & & Reference & & Reference & & Reference & \\
\hline Middle third of esophagus & $1.03(0.95-1.11)$ & 0.517 & $1.15(1.07-1.25)$ & $<0.001$ & $1.03(0.95-1.13)$ & 0.373 & $1.17(1.07-1.27)$ & 0.001 \\
\hline Lower third of esophagus & $0.90(0.84-0.97)$ & 0.007 & $1.13(1.04-1.23)$ & 0.003 & $0.93(0.86-1.01)$ & 0.080 & $1.14(1.04-1.24)$ & 0.004 \\
\hline \multicolumn{9}{|l|}{ TNM Stage } \\
\hline I vs II vs III vs IV & $1.55(1.52-1.58)$ & $<0.001$ & $1.38(1.36-1.41)$ & $<0.001$ & $1.65(1.62-1.69)$ & $<0.001$ & $1.46(1.43-1.49)$ & $<0.001$ \\
\hline \multicolumn{9}{|l|}{ Therapy } \\
\hline Surgery and radiotherapy & Reference & & Reference & & Reference & & Reference & \\
\hline Only surgery & $0.80(0.74-0.86)$ & $<0.001$ & $1.01(0.94-1.03)$ & 0.445 & $0.74(0.68-0.81)$ & $<0.001$ & $0.99(0.91-1.08)$ & 0.817 \\
\hline Only radiotherapy & $2.08(1.84-2.35)$ & $<0.001$ & $1.63(1.44-1.85)$ & $<0.001$ & $2.22(1.95-2.53)$ & $<0.001$ & $1.68(1.48-1.92)$ & $<0.001$ \\
\hline No surgery or radiotherapy & $2.94(2.79-3.09)$ & $<0.001$ & $2.44(2.31-2.57)$ & $<0.001$ & $3.04(2.87-3.21)$ & $<0.001$ & $2.49(2.35-2.64)$ & $<0.001$ \\
\hline
\end{tabular}

$\mathrm{SEER}=$ surveillance, epidemiology and end results; $\mathrm{OS}=$ overall survival; $\mathrm{CSS}=$ cause-specific survival; $\mathrm{ESCC}=$ esophageal squamous cell carcinoma; $\mathrm{EAC}=$ esophageal adenocarcinoma; $\mathrm{TNM}=$ tumor, node and metastasis.

single, widowed, and divorced. As shown in Figure 2A, compared with married patients, divorced patients (HR: 1.23 ; 95\% CI: $1.17-1.30 ; \mathrm{P}<0.0001)$ and single patients (HR: 1.21; 95\% CI: 1.15-1.28; P < 0.0001) had similarly poor prognoses according to the Cox proportional hazards univariate regression model.
Widowed patients had the poorest OS (HR: 1.46; 95\% CI: $1.38-1.55 ; \mathrm{P}<0.0001)$. After controlling all baseline characteristics using the Cox proportional hazards multivariate regression model, divorced patients (HR: 1.20; 95\% CI: 1.14-1.27; P < 0.0001), single patients (HR: 1.22; 95\% CI: 1.15-1.28; 
$\mathrm{P}<0.0001$ ), and widowed patients (HR: 1.25; 95\% CI: $1.17-1.33 ; \mathrm{P}<0.0001)$ all had poorer OS than married patients.

As shown in Figure 2B, compared with married patients, divorced patients (HR: $1.21 ; 95 \%$ CI: 1.14 1.28; $\mathrm{P}<0.0001)$ and single patients (HR: 1.20; 95\% CI: $1.14-1.27 ; \mathrm{P}<0.0001)$ had poorer CSS using the Cox proportional hazards univariate regression model. Widowed patients had the poorest CSS (HR: 1.43; 95\% CI: 1.34-1.52; $\mathrm{P}<0.0001)$. After controlling all baseline characteristics, divorced patients (HR: 1.18; 95\% CI: 1.11-1.25; $\mathrm{P}<0.0001$ ), single patients (HR: 1.20; 95\% CI: $1.13-1.27 ; \mathrm{P}<0.0001$ ), and widowed patients (HR: 1.25; 95\% CI: $1.17-1.34$; $\mathrm{P}<0.0001)$ all had poorer OS than married patients.

\section{Subgroup analysis of the effects of different marital statuses on overall survival and cause- specific survival in the unmatched 15,598 -patient cohort with esophageal cancer according to tumor stage}

Since widowed patients appeared to have more early stage EC, we analyzed whether unmarried status or single, divorced, or widowed status contributed to the poor survival rates in the subgroups of EC patients with different tumor stages according to American Joint
Committee on Cancer (AJCC) staging (sixth edition). Using univariate and multivariate Cox regression analysis, some interesting issues were found. First, unmarried status contributed to poor OS and CSS in each group of patients with different AJCC stage tumors in the univariate and multivariate Cox model $(\mathrm{P}<0.001)$. Second, when the unmarried group was divided into the single, widowed, and divorced groups, the widowed patients had the poorest OS and CSS of the patients with stage I, II, or III EC in the univariate and multivariate Cox model compared with the married, single, and divorced groups. However, in patients with stage IV EC, single patients had the poorest OS and CSS. Detailed information about the OS and CSS of the married patients is shown in Tables 3 and 4. In addition, the subgroup analysis based on different baseline characteristics is shown in Supplementary Tables 1 and 2.

\section{The effects of marital status on overall survival and cause-specific survival in the 5,972-matched patient cohort with esophageal cancer}

Using a 1:1 propensity score matching method, we matched 2,986 unmarried patients with 2,986 married patients. As shown in Supplementary Table 3, the SD between all the baseline clinicopathological factors decreased after matching the data. Using a SD of 0.1 as a cutoff for imbalance, the distribution of sex, race,
A

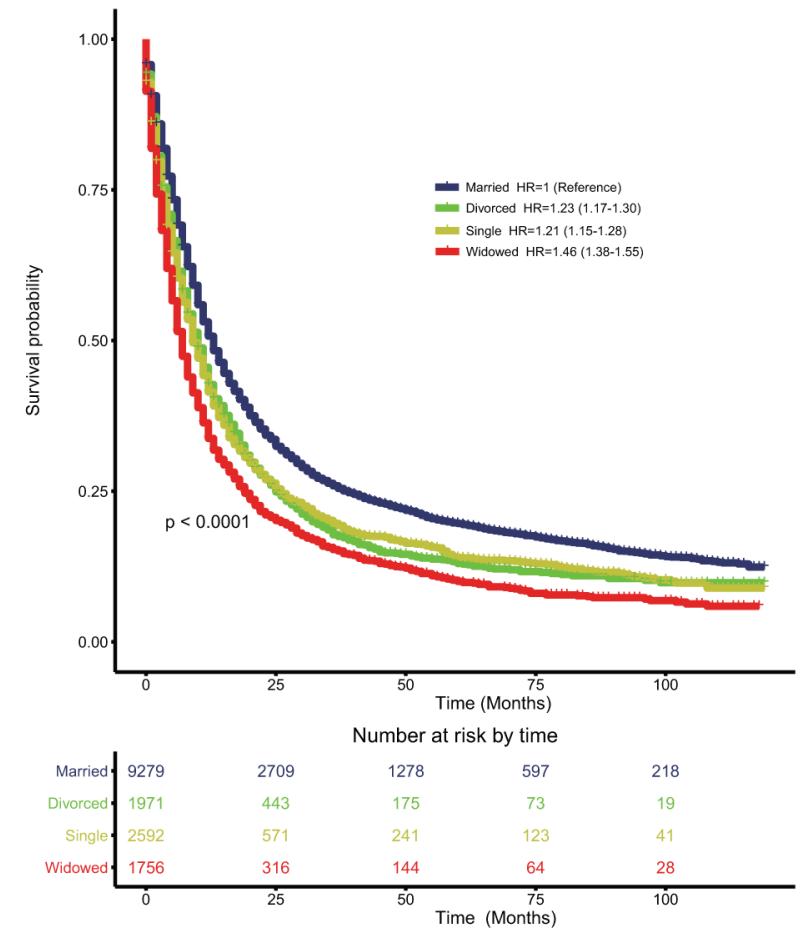

B

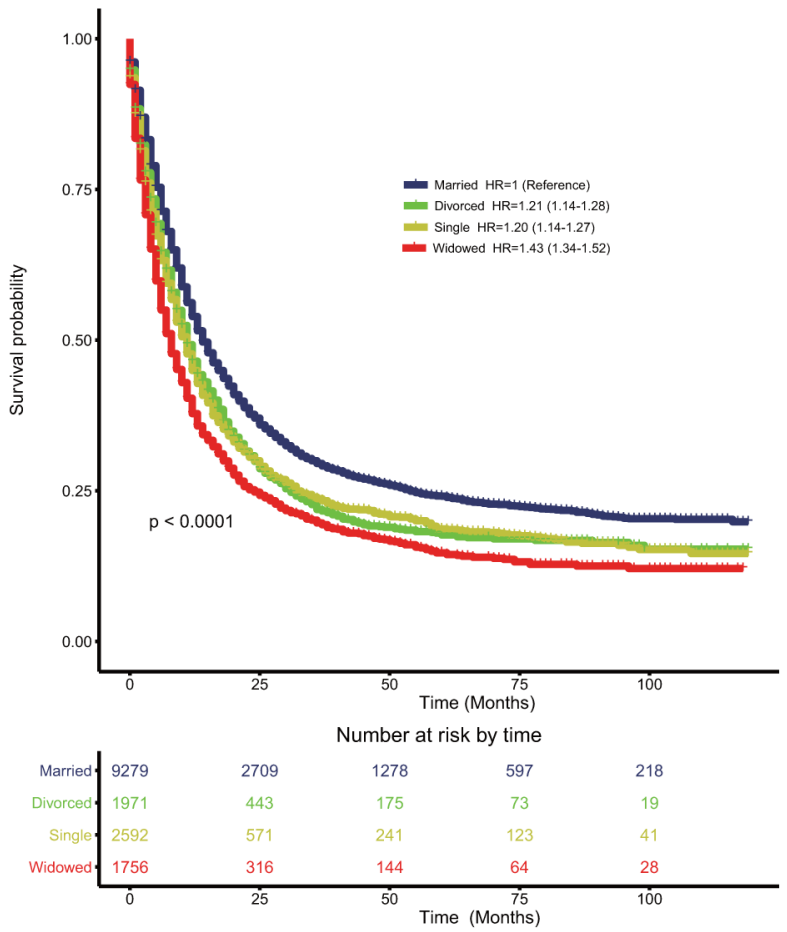

Figure 2: Kaplan-Meier survival plots of the 15,558 patients with esophageal cancers among single, married, widowed, and divorced patients (A) overall survival; (B) esophageal cancer cause-specific survival. 
Table 3: Univariate and multivariate Cox regression analysis of unmarried status compared with married status on OS and esophageal cancer CSS based on different $6^{\text {th }}$ AJCC stage in 15598 unmatched cohort with esophageal cancer

\begin{tabular}{|c|c|c|c|c|c|c|c|c|}
\hline \multirow[t]{3}{*}{ Variable } & \multicolumn{4}{|c|}{ OS } & \multicolumn{4}{|c|}{ CSS } \\
\hline & \multicolumn{2}{|c|}{ Univariate analysis } & \multicolumn{2}{|c|}{ Multivariate analysis } & \multicolumn{2}{|c|}{ Univariate analysis } & \multicolumn{2}{|c|}{ Multivariate analysis } \\
\hline & $\begin{array}{c}\text { HR }(95 \% \\
\text { CI) }\end{array}$ & $\mathbf{P}$ & $\begin{array}{c}\text { HR }(95 \% \\
\text { CI) }\end{array}$ & $\mathbf{P}$ & $\begin{array}{c}\text { HR }(95 \% \\
\text { CI) }\end{array}$ & $\mathbf{P}$ & $\begin{array}{c}\text { HR }(95 \% \\
\text { CI) }\end{array}$ & $\mathbf{P}$ \\
\hline \multicolumn{9}{|l|}{ TNM Stage } \\
\hline \multicolumn{9}{|l|}{ Stage I } \\
\hline Married & Reference & & Reference & & Reference & & Reference & \\
\hline Unmarried & $\begin{array}{c}1.59(1.44- \\
1.76)\end{array}$ & $<0.001$ & $\begin{array}{c}1.27(1.14- \\
1.42)\end{array}$ & $<0.001$ & $\begin{array}{c}1.70(1.52- \\
1.92)\end{array}$ & $<0.001$ & $\begin{array}{c}1.30(1.15- \\
1.47)\end{array}$ & $<0.001$ \\
\hline \multicolumn{9}{|l|}{ Stage II } \\
\hline Married & Reference & & Reference & & Reference & & Reference & \\
\hline Unmarried & $\begin{array}{c}1.27(1.17- \\
1.38)\end{array}$ & $<0.001$ & $\begin{array}{c}1.19(1.09- \\
1.30)\end{array}$ & $<0.001$ & $\begin{array}{c}1.25(1.14- \\
1.37)\end{array}$ & $<0.001$ & $\begin{array}{c}1.17(1.06- \\
1.29)\end{array}$ & 0.002 \\
\hline \multicolumn{9}{|l|}{ Stage III } \\
\hline Married & Reference & & Reference & & Reference & & Reference & \\
\hline Unmarried & $\begin{array}{c}1.36(1.26- \\
1.46)\end{array}$ & $<0.001$ & $\begin{array}{c}1.28(1.18- \\
1.38)\end{array}$ & $<0.001$ & $\begin{array}{c}1.33(1.22- \\
1.43)\end{array}$ & $<0.001$ & $\begin{array}{c}1.25(1.15- \\
1.36)\end{array}$ & $<0.001$ \\
\hline \multicolumn{9}{|l|}{ Stage IV } \\
\hline Married & Reference & & Reference & & Reference & & Reference & \\
\hline Unmarried & $\begin{array}{c}1.22(1.15- \\
1.29)\end{array}$ & $<0.001$ & $\begin{array}{c}1.20(1.13- \\
1.27)\end{array}$ & $<0.001$ & $\begin{array}{c}1.20(1.13- \\
1.27)\end{array}$ & $<0.001$ & $\begin{array}{c}1.18(1.11- \\
1.25)\end{array}$ & $<0.001$ \\
\hline
\end{tabular}

$\mathrm{AJCC}=$ American Joint Committee on Cancer; $\mathrm{OS}=$ overall survival; $\mathrm{CSS}=$ cause-specific survival; $\mathrm{TNM}=$ tumor, node and metastasis.

histology, tumor location, and type of therapy between the two groups reached balance (see Supplementary Table 3).

As shown in the Figure 3, although the HR was not higher after matching the data than before matching the data, unmarried patients still had poorer OS (HR: 1.19; 95\% CI: $1.12-1.25 ; \mathrm{P}<0.0001)$ and CSS (HR: $1.15 ; 95 \%$ CI: $1.09-1.22 ; \mathrm{P}<0.0001)$ than married patients in the univariate Cox analysis. Even after adjusting for these baseline characteristics using multivariate Cox regression (see Supplementary Table 4), unmarried patients still had poorer OS (HR: 1.20; 95\% CI: 1.13-1.27; $\mathrm{P}<0.0001$ ) and CSS (HR: 1.17; 95\% CI: 1.10-1.24; $\mathrm{P}<0.0001$ ) than married patients. To examine the credibility of our conclusions, we conducted additional sensitivity and subgroup analysis. As shown in Figure 4, most of the subgroup analyses showed that unmarried patients had poorer OS and CSS than married patients. Although no significance was reached for some subgroup analyses, such as the analyses of patients of other races for OS and patients with grade I tumors, due to the limited number of patients, there were trends indicating that unmarried status contributed to poorer OS and CSS.

\section{DISCUSSION}

This study was the first to use a systematic PSM method to show that unmarried status was an independent risk factor contributing to poor OS and CSS in EC patients. Unmarried patients had poor OS and CSS regardless of whether the cohort was adjusted for age, sex, race, histology, tumor grade, tumor location, or type of therapy. Even in the matched cohort, unmarried status contributed to the poor survival rate of EC patients. Moreover, different types of unmarried statuses, including single, widowed, and divorced statuses, all contributed to poorer survival rates than married status. Among the unmarried patients, widowed patients were the most likely to die of EC even when adjusting for age, sex, race, tumor stage, tumor grade, tumor location, tumor histology, and type of therapy. 
Table 4: Univariate and multivariate Cox regression analysis of divorced, single and widowed status compared with married status on OS and esophageal cancer CSS based on different $6^{\text {th }}$ AJCC stage in 15598 unmatched cohort with esophageal cancer

\begin{tabular}{|c|c|c|c|c|c|c|c|c|}
\hline \multirow[t]{3}{*}{ Variable } & \multicolumn{4}{|c|}{ OS } & \multicolumn{4}{|c|}{ CSS } \\
\hline & \multicolumn{2}{|c|}{ Univariate analysis } & \multicolumn{2}{|c|}{ Multivariate analysis } & \multicolumn{2}{|c|}{ Univariate analysis } & \multicolumn{2}{|c|}{ Multivariate analysis } \\
\hline & HR $(95 \%$ CI $)$ & $\mathbf{P}$ & HR (95\% CI) & $\mathbf{P}$ & HR $(95 \%$ CI $)$ & $\mathbf{P}$ & HR (95\% CI) & $\mathbf{P}$ \\
\hline \multicolumn{9}{|l|}{ TNM Stage } \\
\hline \multicolumn{9}{|l|}{ Stage I } \\
\hline Married & Reference & & Reference & & Reference & & Reference & \\
\hline Divorced & $1.41(1.19-1.66)$ & $<0.001$ & $1.37(1.16-1.63)$ & $<0.001$ & $1.47(1.22-1.78)$ & $<0.001$ & $1.37(1.13-1.67)$ & $<0.001$ \\
\hline Single & $1.21(1.05-1.41)$ & 0.011 & $1.14(0.98-1.32)$ & 0.100 & $1.24(1.05-1.48)$ & 0.013 & $1.11(0.93-1.32)$ & 0.257 \\
\hline Widowed & $2.29(2.01-2.62)$ & $<0.001$ & $1.36(1.17-1.57)$ & $<0.001$ & $2.55(2.20-2.96)$ & $<0.001$ & $1.45(1.23-1.71)$ & $<0.001$ \\
\hline \multicolumn{9}{|l|}{ Stage II } \\
\hline Married & Reference & & Reference & & Reference & & Reference & \\
\hline Divorced & $1.19(1.05-1.35)$ & 0.007 & $1.18(1.04-1.34)$ & 0.013 & $1.19(1.04-1.37)$ & 0.011 & $1.17(1.02-1.35)$ & 0.025 \\
\hline Single & $1.15(1.02-1.29)$ & 0.023 & $1.17(1.03-1.33)$ & 0.012 & $1.11(0.97-1.26)$ & 0.135 & $1.11(0.97-1.27)$ & 0.130 \\
\hline Widowed & $1.53(1.36-1.73)$ & $<0.001$ & $1.21(1.06-1.39)$ & 0.005 & $1.53(1.34-1.74)$ & $<0.001$ & $1.24(1.07-1.43)$ & 0.005 \\
\hline \multicolumn{9}{|l|}{ Stage III } \\
\hline Married & Reference & & Reference & & Reference & & Reference & \\
\hline Divorced & $1.29(1.16-1.44)$ & $<0.001$ & $1.29(1.15-1.44)$ & $<0.001$ & $1.25(1.11-1.40)$ & $<0.001$ & $1.24(1.10-1.40)$ & $<0.001$ \\
\hline Single & $1.26(1.14-1.40)$ & $<0.001$ & $1.25(1.12-1.39)$ & $<0.001$ & $1.26(1.13-1.40)$ & $<0.001$ & $1.24(1.11-1.39)$ & $<0.001$ \\
\hline Widowed & $1.69(1.49-1.91)$ & $<0.001$ & $1.31(1.14-1.49)$ & $<0.001$ & $1.62(1.41-1.85)$ & $<0.001$ & $1.27(1.10-1.46)$ & 0.001 \\
\hline \multicolumn{9}{|l|}{ Stage IV } \\
\hline Married & Reference & & Reference & & Reference & & Reference & \\
\hline Divorced & $1.16(1.07-1.26)$ & $<0.001$ & $1.16(1.07-1.27)$ & $<0.001$ & $1.13(1.04-1.23)$ & 0.005 & $1.13(1.04-1.24)$ & 0.006 \\
\hline Single & $1.21(1.12-1.30)$ & $<0.001$ & $1.23(1.14-1.33)$ & $<0.001$ & $1.20(1.11-1.30)$ & $<0.001$ & $1.23(1.13-1.33)$ & $<0.001$ \\
\hline Widowed & $1.32(1.20-1.46)$ & $<0.001$ & $1.17(1.06-1.30)$ & 0.003 & $1.30(1.18-1.44)$ & $<0.001$ & $1.15(1.03-1.28)$ & 0.011 \\
\hline
\end{tabular}

AJCC $=$ American Joint Committee on Cancer; $\mathrm{OS}=$ overall survival; $\mathrm{CSS}=$ cause-specific survival; $\mathrm{TNM}=$ tumor, node and metastasis.

Multiple studies have been conducted to show that unmarried patients with gastrointestinal tumors had poorer survival rates than married patients $[3,5$, 6, 14-19], but these studies included limited evidence about EC patients. Two Swedish population-based cohorts investigated the role of marital status in the survival rates of EC patients but reached contractionary conclusions. Brusselaers et al. examined 606 EC patients and divided marital status into married, remarried, never married, and previously married statuses [10]. This study found no evidence of better five-year survival rates in married patients than in unmarried patients, though this conclusion can likely be attributed to the limited number of patients included in the study. Another Swedish study involved all Swedish residents aged 30-84 years in 1990-2007 and analyzed the role of sociodemographic and geographical factors, including marital status, and produced positive results that showed that married residents had better EC survival rates than unmarried residents [9]. However, this study could not adjust for the clinicopathological factors of EC because it was based on the Causes of Death Register. Therefore, the effect of marital status on the EC survival rate should be considered cautiously. One large well-designed, comprehensive populationbased study analyzed cancers associated with the ten leading causes of cancer-related death, including EC, and found that unmarried status contributed to poor survival rates in all ten cancers [3]. However, tumor grade, location of EC, tumor histology, and more 
A

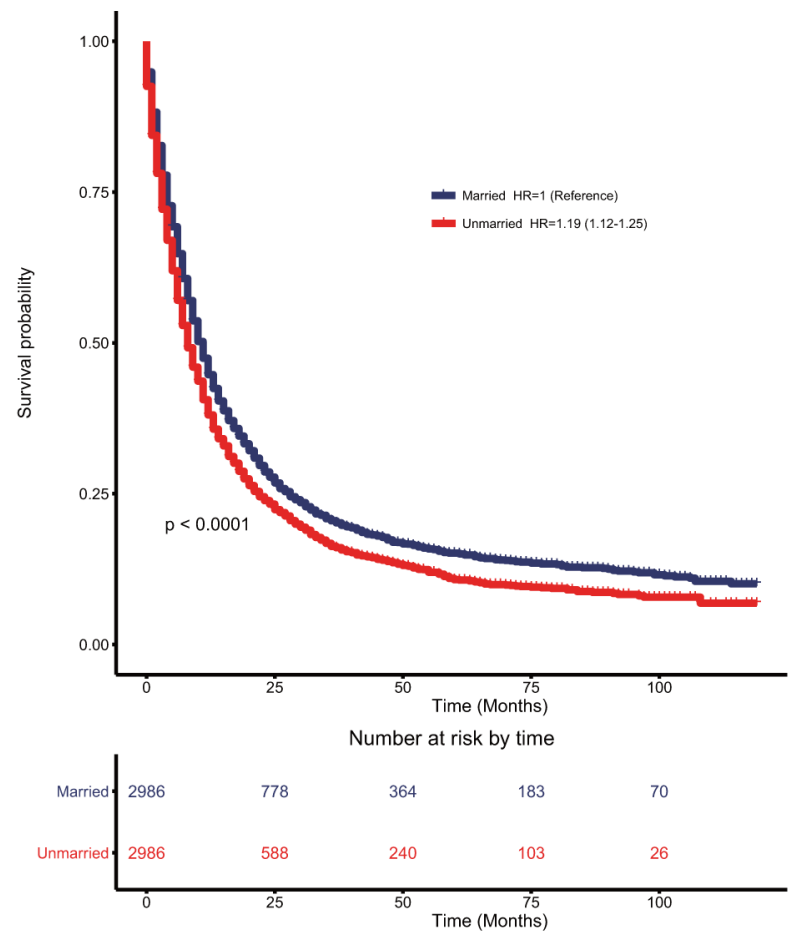

B

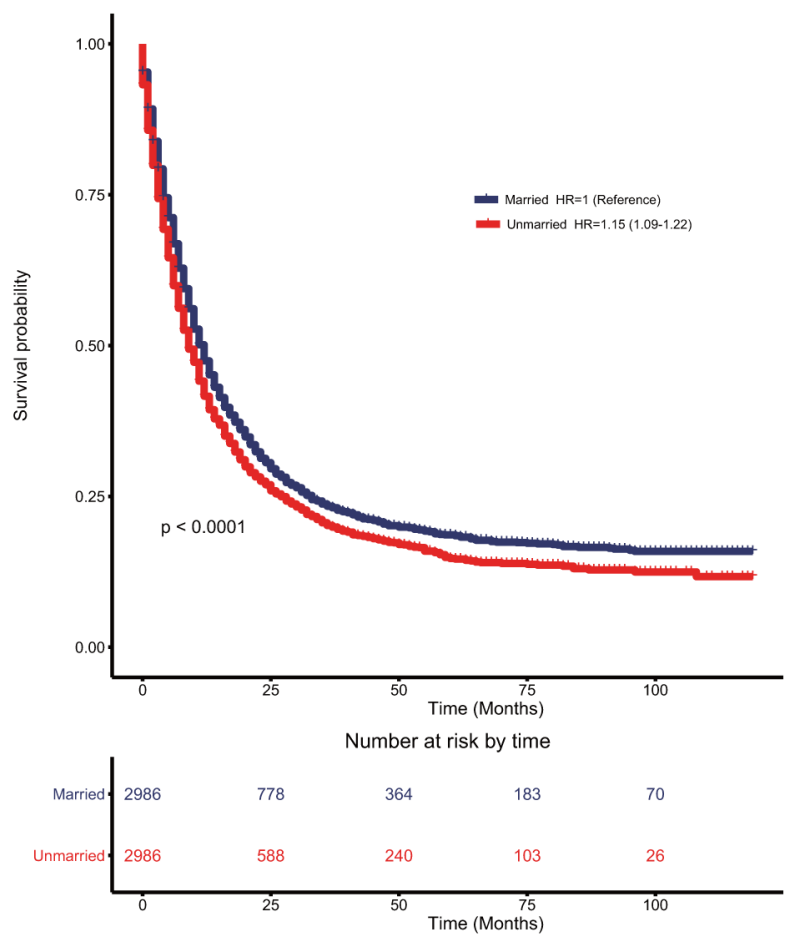

Figure 3: Kaplan-Meier survival plots in patients with esophageal cancers in the matched cohort with 2,986 unmarried and 2,986 married patients according to marital status (A) overall survival; (B) esophageal cancer cause-specific survival.

A

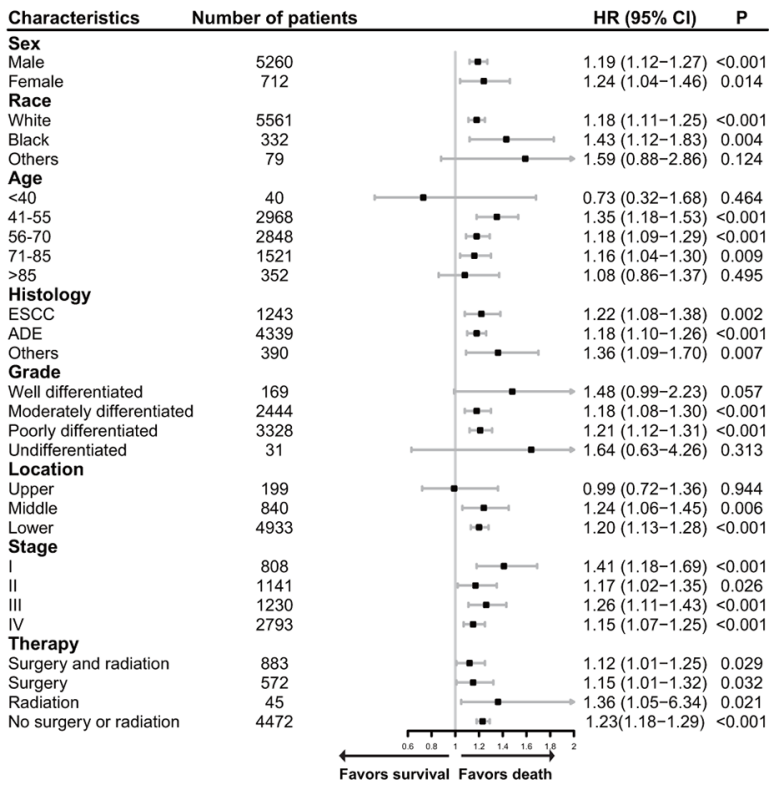

B

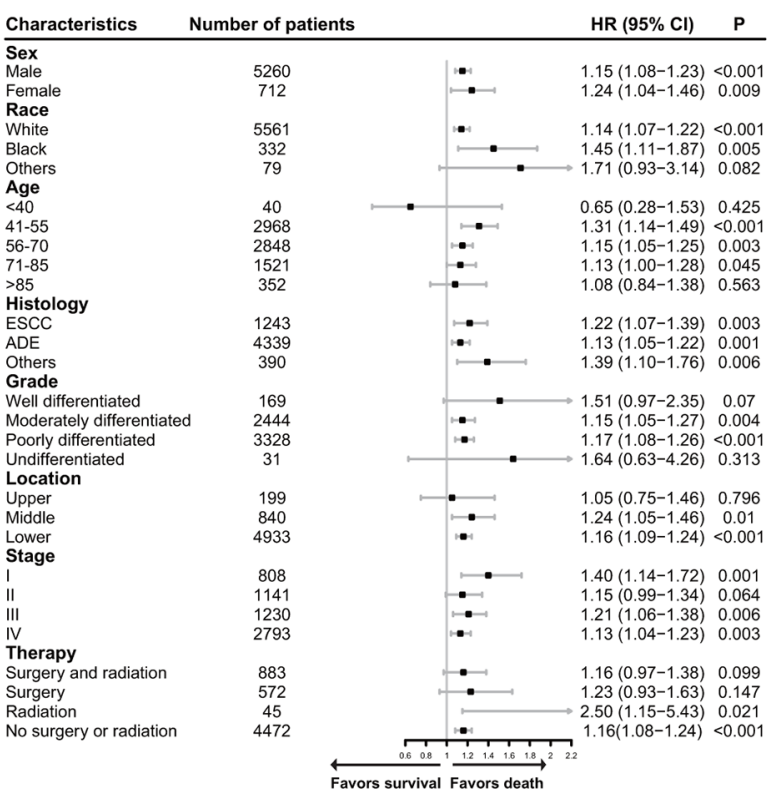

Figure 4: Forest plot presenting the contribution of unmarried status compared with that of married status to the survival rates of patients in the subgroups of the matched cohort according to different clinicopathological factors (A) overall survival; (B) esophageal cancer causespecific survival. HR $>1$ with $\mathrm{P}<0.05$ meant that unmarried status contributed significantly to poorer survival than married status. 
detailed therapy information that may affect the survival rate of EC patients were not included for analysis in this study.

In accordance with Aizer's study, the unmarried patients examined by the present study received less therapy than married patients. However, unmarried patients had percentages of stage I, II, III, and IV tumors that were comparable with that of married patients, with a SD of 0.049 , which differed from Aizer's study. This difference may be because metastatic tumors were considered late-stage tumors in Aizer's study, but TNM stage was used in our study. Unlike Aizer's study, we analyzed the role of different subdivisions of unmarried statuses in the survival rate of EC patients and found that widowed patients had the poorest survival rate of the overall EC population. In addition, we used PSM, which is an effective tool to address selection and residual biases $[20,21]$, to analyze relationships further to support our conclusions that being unmarried contributes to poor EC survival rates. Interestingly, widowed patients, who had the poorest survival rates, were more likely to be diagnosed at an early stage $(22.89 \%)$ compared with $14.85 \%, 16.11 \%$, and $13.50 \%$ in single, married, and divorced patients, respectively, which were rates similar to those found in gastric cancer patients [6]. In addition, our study explored whether the contributions of unmarried status and even the different subdivisions of unmarried statuses persisted in subgroups of EC patients with different stages of cancer. Interestingly, subgroup analysis showed that widowed patients had the poorest survival rate for stage I, II, and III EC, but single patients had the poorest survival rate for stage IV EC.

These results indicate that some latent risk factors also play an important role in the poor survival rate of unmarried patients. These risk factors include social factors, which could be explained hypothetically from psychosocial and socioeconomic perspectives. Having a support system that includes emotional support is one factor that might affect cancer survival rates from a psychosocial perspective, and financial support is one factor that might affect these rates from a socioeconomic perspective. From a socioeconomic perspective, patients with cancer usually have financial dilemmas due to the high cost of cancer treatments. Having another source of income could help lighten this financial burden [22, 23]. Without enough financial support to cover their EC treatments, some unmarried patients may refuse to receive the care they need, which was proved in our study. However, the income of a married patient's partner could help alleviate the burden of these medical expenses.

From a psychosocial perspective, spouses could also help married patients receive the care they need and encourage married patients to be positive about the outcome of their EC, but this type of support is a privilege that unmarried patients lack. In addition, being unmarried has been associated with a higher incidence of depression among cancer patients [14, 24-26]. Studies have shown that depressive disorders could affect nearly $26 \%$ of patients, with some of these patients refusing to be treated actively, and the symptoms of this depression might even persist long after therapy or could reappear upon cancer recurrence $[27,28]$. In our study, after using PSM, the differences between the tumor characteristics and the extent of treatment, such as tumor grade, therapy options, and tumor stage at diagnosis, nearly disappeared, but unmarried status still contributed to poorer prognoses. This result further supported previous results that suggested that unmarried status affects survival on a psychosocial level.

Since psychosocial support influences the prognoses of EC patients, marital status should be taken into consideration, especially widowed status, which carries the highest risk of death. Unmarried EC patients should be provided with more psychosocial care. For example, counseling could enable unmarried patients to discuss their EC diagnosis and therapy options. Meanwhile, barriers in seeking optimal care could be reduced [29]. In addition, social workers could also help unmarried patients during their initial EC diagnosis, treatment, and follow-ups with adequate companion and life assistance [30, 31].

Several limitations of our study should be discussed. First, the marital status analyzed in our study was defined at the initial diagnosis of each tumor and might have changed during follow-up periods. We could not adjust this bias because the SEER database only included information about marital status at the time of diagnosis. Second, detailed information about the patients' therapies, such as chemotherapy and therapy quality, was not provided. Third, only legally married patients were classified as married, which meant that cohabitating patients were not recorded as married, biasing our results. According to the 2010 US Census, approximately 90 million unmarried individuals were cohabitating with other people, whereas only about 30 million (25\%) lived alone. Fourth, patients with unknown clinicopathological characteristics were excluded from our analysis, causing selection bias. However, this problem was addressed to some extent by the use of PSM in our study. Fifth, our matching procedure resulted in the inclusion of only $38 \%(5,972 / 15,598)$ of the original cohort.

Despite the inevitable limitations of our study, our results demonstrated that unmarried status could contribute to the poor survival rates of EC patients, and these conclusions were further confirmed by PSM. Furthermore, after unmarried status was divided into three subgroups, we showed that all single, widowed, and divorced patients had poorer prognoses than married patients. Among these unmarried subgroups, widowed patients had the worst prognoses, which suggests that these patients need more counseling and comprehensive case management. 


\section{METHODS}

\section{Data source}

Data was obtained from the SEER program, which is sponsored by the National Cancer Institute. The SEER program includes data from 18 population-based cancer registries from 1973 to 2013, which represents approximately $30 \%$ of US population. The program collects data about cancer stage, grade, therapy, incidence, and demographic information, such as age, sex, race, and marital status. The current dataset used for this analysis was based on the Incidence-SEER 18 Register Research Data + Hurricane Katrina Impacted Louisiana Cases, Nov2015 Sub (1973-2013 varying). Since the SEER database did not include personal identifying information, informed consent was not required in our study. However, permission to access SEER database was approved using the private SEER ID (13526-Nov2015). This study was approved by our institutional review board at Ren Ji Hospital.

\section{Inclusion and exclusion criteria}

Patients with esophageal primary cancers were identified with the following inclusion criteria: 1) site recode ICD-O-3/WHO 2008 (International Classification of Diseases for Oncology, 3rd edition) was restricted to the "Esophagus"; 2) the year of diagnosis of the cancers ranged from 2004 to 2012; 3) EC was the only single cancer or was the first tumor of more than one primary tumors; 4) all relevant factors contributing to survival should be known, including age, sex, race, histology, TNM stage, tumor grade, whether surgery was performed, location of the primary cancer, and whether radiotherapy was performed; and 5) the cause of death and survival time were both known.

\section{Variables}

The main independent variable of interest was each patient's marital status at the initial diagnosis of the primary cancer. Marital status was redefined as married or unmarried (including single, widowed, divorced, separated, or domestic partner). For further analysis, we also divided marital status into four groups: married, divorced (including separated and divorced), single (including single, unmarried, and domestic partner) and widowed. Race was classified as White, Black, or other (including American Indian/AK Native and Asian/ Pacific Islander) and excluded unknown race. Age was presented with five groups: $<40,41-55,56-70,71-85$, $>85$. Histology was reclassified as esophageal squamous cell carcinoma (ESCC), EAC, or other since ESCC and EAC are two main types of esophageal cancers. Tumor grade was defined as well differentiated, moderately differentiated, poorly differentiated, or undifferentiated, excluding unknown grade. We divided tumor location into three groups, including the upper third of the esophagus, the middle third of the esophagus, and the lower third of the esophagus. TNM stage was defined as stage I, II, III, or IV using the AJCC Cancer Staging Manual (sixth edition). Type of therapy was defined as whether surgery or radiotherapy was performed, including no surgery or radiotherapy, only surgery, only radiotherapy, or surgery and radiotherapy, because whether surgery or radiotherapy were performed could contribute to the survival outcomes of patients.

The main outcomes were OS and CSS. CSS was defined as a net survival measure representing the survival of a specific cause of death, excluding other causes of death, according the SEER database. In this study, CSS was defined as death due to EC, with individuals who died of causes other than EC being censored.

\section{Statistical analysis}

The clinical characteristics of the patients with EC were presented with descriptive statistics. The categorical variable was presented with the proportion of patients. The continuous variable was shown as mean \pm standard deviation. A Kaplan-Meier plot was used to show OS and CSS. The comparison between patients with different marital statuses was performed using the log-rank test. In addition, HR was calculated for known prognostic factors, including age, sex, race, therapy, histology, TNM stage, tumor grade, tumor location, and marital status, using the univariate Cox proportional hazard model and the multivariate Cox proportional hazard model.

In order to reduce imbalance, we also used PSM to carry out a matched case-control analysis [20]. First, the propensity scores were calculated for marital status (married and unmarried) for each of the 15,598 patients using a nonparsimonious multivariable logistic regression model. In this model, marital status was used as the dependent variable, with all recorded variables shown in Table 1 included as covariates. Second, using an SPSS matching macro, we matched married and unmarried patients who had very similar propensity scores. Third, after matching, all baseline covariates between married and unmarried patients before and after propensity scores were matched using SD $<0.1$, suggesting that these covariates between married and unmarried patients were well balanced. Fourth, we used Kaplan-Meier survival analyses to compare the OS and CSS of married and unmarried patients. In addition, the Cox univariate proportional hazards model and the Cox multivariate proportional hazards model were used to calculate the HR of marital status on OS and CSS, respectively. Fifth, sensitivity analyses and subgroup analyses were conducted using Cox multivariate proportional hazards model to determine the reliability of the association between marital status and OS or CSS [32, 33]. 
All statistical tests were evaluated using a twotailed $95 \% \mathrm{CI}$, and two-side $\mathrm{P}<0.05$ was set for statistical significance. All data analyses were performed using the statistical software package SPSS for Windows, version 17 (SPSS Inc, Chicago, IL, USA).

\section{Abbreviations}

EC: esophageal cancer; SEER: the surveillance, epidemiology and end results; OS: overall survival; CSS: cancer specific survival; HR: hazard ratio; PSM: propensity score matching; TNM: tumor, node and metastasis; SD: standard difference; CI: confidence level; ESCC: esophageal squamous cell carcinoma; EAC: esophageal adenocarcinoma

\section{Author contributions}

QWZ, XLL, LMT, CYT and CHZ contributed equally to this work. QWZ, XLL, ZZG and XBL conceived and designed the study. QWZ and LMT, ZXT performed the analyses. CYT and CHZ prepared all figures and tables. QWZ, XLL, LMT, CYT, and CHZ wrote the main manuscript. All authors reviewed the manuscript.

\section{ACKNOWLEDGMENTS}

The authors acknowledge the efforts of the SEER program in the creation of the SEER database.

\section{CONFLICTS OF INTEREST}

The authors don't declare any conflict of interest.

\section{FUNDING}

This study was supported by Incubating Program for Clinical Research and Innovation of Ren Ji Hospital, School of Medicine, Shanghai Jiao Tong University (Grant No. PYMDT-001)

\section{REFERENCES}

1. Siegel RL, Miller KD, Jemal A. Cancer statistics, 2017. CA Cancer J Clin. 2017; 67:7-30.

2. Njei B, McCarty TR, Birk JW. Trends in esophageal cancer survival in United States adults from 1973 to 2009: a SEER database analysis. J Gastroenterol Hepatol. 2016; 31:1141-1146.

3. Aizer AA, Chen MH, McCarthy EP, Mendu ML, Koo S, Wilhite TJ, Graham PL, Choueiri TK, Hoffman KE, Martin $\mathrm{NE}, \mathrm{Hu}$ JC, Nguyen PL. Marital status and survival in patients with cancer. J Clin Oncol. 2013; 31:3869-3876.

4. He XK, Lin ZH, Qian Y, Xia D, Jin P, Sun LM. Marital status and survival in patients with primary liver cancer.
Oncotarget. 2016. doi: 10.18632/oncotarget.11066. [Epub ahead of print]

5. Li Q, Gan L, Liang L, Li X, Cai S. The influence of marital status on stage at diagnosis and survival of patients with colorectal cancer. Oncotarget. 2015; 6:7339-7347. doi: 10.18632/oncotarget.3129.

6. Shi RL, Chen Q, Yang Z, Pan G, Zhang Z, Wang W, Liu S, Zhang D, Jiang D, Liu W. Marital status independently predicts gastric cancer survival after surgical resection--an analysis of the SEER database. Oncotarget. 2016; 7:1322813235. doi: 10.18632/oncotarget.7107.

7. Wang XD, Qian JJ, Bai DS, Li ZN, Jiang GQ, Yao J. Marital status independently predicts pancreatic cancer survival in patients treated with surgical resection: an analysis of the SEER database. Oncotarget. 2016; 7:24880-24887. doi: 10.18632/oncotarget.7107.

8. Uchino BN. Social support and health: a review of physiological processes potentially underlying links to disease outcomes. J Behav Med. 2006; 29:377-387.

9. Ljung R, Drefahl S, Andersson G, Lagergren J. Sociodemographic and geographical factors in esophageal and gastric cancer mortality in Sweden. PLoS One. 2013; 8:e62067.

10. Brusselaers N, Mattsson F, Johar A, Wikman A, Lagergren P, Lagergren J, Ljung R. BMJ Open. 2014; 4:e005418.

11. Kravdal H, Syse A. Changes over time in the effect of marital status on cancer survival. BMC Public Health. 2011; 11:804.

12. Goodwin JS, Hunt WC, Key CR, Samet JM. The effect of marital status on stage, treatment, and survival of cancer patients. JAMA. 1987; 258:3125-3130.

13. Kravdal O. (2000). Social inequalities in cancer survival. Population Studies, pp. 1-18.

14. Tsunoda A, Nakao K, Hiratsuka K, Yasuda N, Shibusawa M, Kusano M. Anxiety, depression and quality of life in colorectal cancer patients. Int J Clin Oncol. 2005; $10: 411-417$.

15. Jin JJ, Wang W, Dai FX, Long ZW, Cai H, Liu XW, Zhou Y, Huang H, Wang YN. Marital status and survival in patients with gastric cancer. Cancer Med. 2016; 5:1821-1829.

16. Qiu M, Yang D, Xu R. Impact of marital status on survival of gastric adenocarcinoma patients: results from the surveillance epidemiology and end results (SEER) database. Sci Rep. 2016; 6:21098.

17. Zhang J, Gan L, Wu Z, Yan S, Liu X, Guo W. The influence of marital status on the stage at diagnosis, treatment, and survival of adult patients with gastric cancer: a populationbased study. Oncotarget. 2016; 8:22385-22405. doi: 10.18632/oncotarget.7399.

18. Johansen C, Schou G, Soll-Johanning H, Mellemgaard A, Lynge E. Influence of marital status on survival from colon and rectal cancer in Denmark. Br J Cancer. 1996; 74:985-988. 
19. Wang L, Wilson SE, Stewart DB, Hollenbeak CS. Marital status and colon cancer outcomes in US surveillance, epidemiology and end results registries: does marriage affect cancer survival by gender and stage? Cancer Epidemiol. 2011; 35:417-422.

20. D'Agostino RB Jr. Propensity score methods for bias reduction in the comparison of a treatment to a nonrandomized control group. Stat Med. 1998; 17:2265-2281.

21. Aronow HD, Topol EJ, Roe MT, Houghtaling PL, Wolski KE, Lincoff AM, Harrington RA, Califf RM, Ohman EM, Kleiman NS, Keltai M, Wilcox RG, Vahanian A, et al. Effect of lipid-lowering therapy on early mortality after acute coronary syndromes: an observational study. Lancet. 2001; 357:1063-1068.

22. O’Neill CB, Atoria CL, O’Reilly EM, LaFemina J, Henman $\mathrm{MC}$, Elkin EB. Costs and trends in pancreatic cancer treatment. Cancer. 2012; 118:5132-5139.

23. Baker MS, Kessler LG, Urban N, Smucker RC. Estimating the treatment costs of breast and lung cancer. Med Care. $1991 ; 29: 40-49$

24. Schlegel RJ, Manning MA, Molix LA, Talley AE, Bettencourt BA. Predictors of depressive symptoms among breast cancer patients during the first year post diagnosis. Psychol Health. 2012; 27:277-293.

25. Bodurka-Bevers D, Basen-Engquist K, Carmack CL, Fitzgerald MA, Wolf JK, de Moor C, Gershenson DM. Depression, anxiety, and quality of life in patients with epithelial ovarian cancer. Gynecol Oncol. 2000; 78:302-308.
26. Alacacioglu A, Yavuzsen T, Dirioz M, Yilmaz U. Quality of life, anxiety and depression in Turkish breast cancer patients and in their husbands. Med Oncol. 2009; 26:415-419.

27. Miovic M, Block S. Psychiatric disorders in advanced cancer. Cancer. 2007; 110:1665-1676.

28. Honda K, Goodwin RD. Cancer and mental disorders in a national community sample: findings from the national comorbidity survey. Psychother Psychosom. 2004; 73:235-242.

29. Marcus AC, Garrett KM, Cella D, Wenzel L, Brady MJ, Fairclough D, Pate-Willig M, Barnes D, Emsbo SP, Kluhsman BC, Crane L, Sedlacek S, Flynn PJ. Can telephone counseling post-treatment improve psychosocial outcomes among early stage breast cancer survivors? Psychooncology. 2010; 19:923-932.

30. Cwikel JG, Behar LC. Organizing social work services with adult cancer patients: integrating empirical research. Soc Work Health Care. 1999; 28:55-76.

31. Sharp JW, Blum D, Aviv L. Elderly men with cancer: social work interventions in prostate cancer. Soc Work Health Care. 1993; 19:91-107.

32. Love T. Using propensity scores: stratification, adjustment, sensitivity and strategies. 2005: http:/www.chrp.org/ propensity/.

33. Rubin D. On principles for modeling propensity scores in medical research. Pharmacoepidemiol Drug Saf. 2004; 13:855-857. 